\title{
CMTM1_v17 is a novel potential therapeutic target in breast cancer
}

\author{
JING WANG ${ }^{1,2^{*}}$, GUOYING ZHANG ${ }^{1,3^{*}}$, YINGMEI ZHANG ${ }^{1,3}$, YANG LUO ${ }^{1,3}$, \\ QUANSHENG SONG ${ }^{1,3}$, XIAOYAN QIU ${ }^{1,3}$, XIAONING MO $\mathrm{MO}^{1,3}$ and LU WANG ${ }^{1,3}$
}

\begin{abstract}
${ }^{1}$ Department of Immunology, Center for Human Disease Genomics, School of Basic Medical Sciences, Health Science Center, Peking University, Beijing 100191; ${ }^{2}$ Department of Clinical Laboratory, Beijing Shijitan Hospital, Capital Medical University, Beijing 100038; ${ }^{3}$ Key Laboratory of Medical Immunology, Ministry of Health, School of Basic Medical Science, Peking University, Beijing 100191, P.R. China
\end{abstract}

Received March 25, 2014; Accepted May 12, 2014

DOI: 10.3892/or.2014.3429

\begin{abstract}
Chemokine-like factor $(C K L F)$-like MARVEL transmembrane domain-containing 1 (CMTMI) consists of at least 23 alternatively spliced isoforms designated $C M T M 1_{-}$ v1-v23. In the present study, we detected CMTM1_v17 expression in multiple human normal and tumor tissues and found that CMTM1_v17 was highly expressed in testis and many tumor tissues including breast tumor. The overexpression of CMTM1_v17 in the breast cancer cell line MDA-MB-231 promoted cell proliferation and resistance to tumor necrosis factor- $\alpha$ (TNF- $\alpha$ )-induced apoptosis. Moreover, siRNA-mediated silencing of $C M T M 1$ v17 sensitized MDA-MB-231 cells to TNF- $\alpha$-induced apoptosis. We propose that CMTM1_v17 may be a novel potential target for therapy in breast cancer patients. The present study provides insight into a novel mechanism by which CMTM1_v17 enhances cellular proliferation and abrogates TNF- $\alpha$-induced apoptosis. These findings also have implications for clinical practice as they highlight the potential for therapeutic targeting of CMTM1_v17 for the treatment of breast and other cancers in which CMTM1_v17 impacts cellular proliferation and survival.
\end{abstract}

\section{Introduction}

Chemokine-like factor $(C K L F)$ was first isolated from PHA-stimulated U937 cells (1). Since then, eight novel genes of the $C K L F$ family have been identified through BLAST searches in combination with expressed sequence tag assembly and have been experimentally validated. They

Correspondence to: Dr Lu Wang, Department of Immunology, Center for Human Disease Genomics, Peking University, 38 Xueyuan Road, Beijing 100191, P.R. China

E-mail: wanglu@bjmu.edu.cn

${ }^{*}$ Contributed equally

Key words: CMTM1_v17, therapeutic target, breast cancer, tumor necrosis factor- $\alpha$, apoptosis have been designated as chemokine-like factor superfamily ( $C K L F S F)$ members and have been approved by the HUGO Gene Nomenclature Committee (2). In 2005, CKLFSF1-8 were renamed $C K L F$-like MARVEL transmembrane domain containing 1-8 (CMTM1-8). CKLF and CMTM1-8 represent a novel protein family linking the chemokine and TM4SF families that may play multiple roles in a wide range of physiological and pathological processes. $C K L F 1$ has chemotactic effects on a wide spectrum of leukocytes, while $C K L F 2$ stimulates the proliferation and differentiation of $\mathrm{C} 2 \mathrm{C} 12$ cells (3). Rat and mouse $c k l f$ have similar RNA splice forms and functions as human $C K L F(4,5)$.

Our laboratory cloned and characterized $C M T M 1$, which is highly expressed in testis tissue. The CMTMl gene consists of seven exons and six introns. Most of the intron-exon boundaries agreed with the GT/AG rule and two alternative transcription start sites, $1 \mathrm{~A}$ and $1 \mathrm{~B}$, were identified. Site $1 \mathrm{~A}$ predicted a complete open reading frame ORF1. Site 1B, located inside the putative exon 1, predicted a shorter open reading frame ORF2. cDNA sequencing revealed that CMTM1 contains at least 23 alternatively spliced isoforms, which were designated CMTM1_v1-v23 (suggested by the HUGO Gene Nomenclature Committee). The proteins of CMTM1_v1-16 are encoded by ORF1 and ORF2 encodes CMTM1_v17-23.

CMTM1 is most abundant in spermatocytes of human testicular tissues (6) and is highly upregulated and greatly susceptible to the effect of hrIL-30 in PC 3 cells (7). CMTM2 is a secreted protein that may be functionally relevant during spermatogenesis (8). CMTM8 is a novel negative regulator of epidermal growth factor-induced signaling via facilitation of ligand-induced receptor endocytosis and subsequent desensitization (9). CMTM3 is highly conserved and highly expressed in both the immune system and the male reproductive system (10). It was reported that CMTM3-5 (11-13) and CMTM7 (14) all exhibited inhibitory effects on the growth of tumor cells. However, the functions of another member of the CKLFSF family, CMTM6, are largely undefined.

In the present study, we found that $C M T M 1$ _v17 was highly expressed in human testis and many human tumor tissues, and cell lines but was almost undetectable in human normal tissues. MDA-MB-231 breast cancer cells overexpressing 
CMTM1_v17 had enhanced proliferation and resisted tumor necrosis factor- $\alpha$ (TNF- $\alpha$ )-induced apoptosis. We suggest that CMTM1_v17 may be a novel potential therapeutic target in breast cancer patients and it may also be a novel potential cancer/testis antigen.

\section{Materials and methods}

Cell culture and transfection. The human cell lines HEK293T (a gift from T. Matsuda, Japan), HEK293, MDA-MB-231, MCF-7, HepG2, SGC7901, AGS, Caov3, HeLa and H1299 were cultured in Dulbecco's modified Eagle's medium (DMEM; Life Technologies, USA) containing $10 \%$ fetal bovine serum (FBS; HyClone, USA), $100 \mathrm{U} / \mathrm{ml}$ penicillin, and $100 \mu \mathrm{g} / \mathrm{ml}$ streptomycin at $37^{\circ} \mathrm{C}$ in a humidified incubator with $5 \% \mathrm{CO}_{2}$. The suspension cell lines K562, Raji and U937 were maintained in RPMI-1640 medium supplemented with FBS and antibiotics as above. MDA-MB-231 cells were plated in fresh culture medium prior to initiating experiments. The indicated amount of siRNA plus plasmid or plasmid alone was transfected into MDA-MB-231 cells using Lipofectamine 2000 (Invitrogen, USA) according to the manufacturer's protocol.

cDNA preparation and real-time $q P C R$. cDNA preparations of normal tissues were purchased from Clontech (Mountain View, CA, USA). Tumor tissues were gifts from Beijing Shijitan Hospital. Three pathologists evaluated all the tissues to establish the presence of tumors. Tumor tissues were homogenated and pellet cells were suspended in TRIzol reagent (Invitrogen) for the isolation of total RNA according to the manufacturer's instructions. cDNA was synthesized from $3 \mu \mathrm{g}$ of total RNA using the ThermoScript ${ }^{\mathrm{TM}}$ RT-PCR System (Invitrogen). We prepared cDNA from multiple cell lines. The resulting cDNA products were used to amplify the fragment of CMTM1_v17 (forward, 5'-ATG TTG AAG ATC CTG AGA CT-3' and reverse, 5'-CAA TGT AAA TAG GTC AGC AA GTG GTG-3') by real-time qPCR using the Power SYBR-Green PCR Master Mix on a AB7500 System (both from Applied Biosystems, USA) as follows: $95^{\circ} \mathrm{C}$ for $30 \mathrm{sec}$, 40 cycles of $95^{\circ} \mathrm{C}$ for $15 \mathrm{sec}, 60^{\circ} \mathrm{C}$ for $1 \mathrm{~min}$. The mRNA levels of CMTM1_v17 were normalized using GAPDH mRNA levels. Tissues that were utilized for RNA or protein extraction were frozen in liquid nitrogen immediately after harvesting. All samples were obtained from patients with their informed consent and the approval of the Ethics Committee.

cDNA cloning and vector construction. The CMTM1_v17 gene was amplified from a cDNA library of the human breast cancer cell line MDA-MB-231 by PCR using the primers: forward, 5'-ATG TTG AAG ATC CTG AGA CT-3' and reverse, 5'-GCA CGT GTC TGT CGA ATC GCT-3'. The purified PCR product was ligated into the pGEM-T Easy vector (Promega, Madison, WI, USA) and the insert was released by EcoRI digestion and ligated into the EcoRI site of pcDNA.3.1/myc-His(-)B (Invitrogen) to construct the plasmid pcDB/CMTM1_v17. The reverse primer (5'-TCGGATCCACGTCTCGTAAAA-3') was fused with a C-terminal GFP tag in pEGFP-N1 (Clontech) vector by BamHI to construct the plasmid CMTM1_v17-EGFP. A C-terminal human CMTM1_v17 cDNA fragment encoding a 31 amino acid hydrophilic region was amplified from the human breast cancer cell line MDA-MB-231 cDNA library using the primers forward, 5'-GAA AAG ATT CCT GGG AGT CG-3' and reverse, 5'-GCA CGT GTC TGT CGA ATC GCT-3'. The PCR product was cloned into pGEX-4T-2 (Pharmacia, USA) for expression in E. coli. All clones were confirmed by sequencing. All plasmids were purified using Qiagen Plasmid kit (Qiagen, Germany) columns.

Confocal microscopy. To determine the subcellular localization of CMTM1_v17, HEK293T cells transfected with control EGFP or CMTM1_v17-EGFP were fixed in 4\% paraformaldehyde for $15 \mathrm{~min}$ and permeabilized with $0.2 \%$ Triton X-100 for $1 \mathrm{~h}$ at room temperature. Cells were rinsed with phosphatebuffered saline (PBS) and stained with DAPI for nuclear visualization. Fluorescence was detected by confocal microscopy (Leica TCS SP5; Confocal System, Germany).

Synthesis of CMTM1_v17 small interfering RNAs. Human CMTM1_v17 double-stranded small interference RNA was synthesized from GeneChem Corporation (Shanghai, China). The sense oligonucleotide, 5' ACC ACU UGC UGA CCU AUU UdTdT and the antisense oligonucleotide, 5' AAA UAG GUC AGC AAG UGG UdTdT were utilized.

Antibody preparation. The GST fusion protein was expressed and purified as described in the GST Gene Fusion System manual (Pharmacia). Polyclonal anti-CMTM1_v17 antiserum was raised in rabbits immunized with the purified GST fusion protein. Immunoreactive serum was identified by ELISA and western blotting (data not shown). The GST tag of the fusion protein was removed with thrombin and the purified antigen was coupled to a chromatographic matrix (Sepharose 4B; Pharmacia). The column was used to purify highly-specific, polyclonal anti-CMTM1_v17 antibodies from the antiserum.

Western blotting. Lysates were harvested from primary tissues and cell lines. Protein lysates $(30-50 \mu \mathrm{g})$ were separated by SDS-PAGE and transferred to nitrocellulose membranes. After transferring, the membranes were blocked in 5\% non-fat dry milk in TBS-T for $2 \mathrm{~h}$ at room temperature followed by incubation overnight at $4^{\circ} \mathrm{C}$ with primary antibodies that were specific for CMTM1_v17, phospho-IKK, IKK $\beta$ and I $\kappa \mathrm{B} \alpha$ (1:1,000; Cell Signaling). Blots were washed with TBS-T and then incubated at room temperature for $1 \mathrm{~h}$ with the appropriate IRDye $^{\text {TM }}$ 800-conjugated secondary antibodies (LI-COR Biosciences, USA). Immunocomplexes were detected using Odyssey Infrared Imager System (LI-COR Biosciences). Membranes were re-probed with $\beta$-actin monoclonal antibody to confirm equal loading.

Tissue microarrays and immunohistochemistry. Three tissue microarray slides (lot nos. CC08-02-004, CC08-11-001 and CC08-11-002) containing tumor, normal and non-cancerous specimens were purchased from Cybrdi (Xi'an, Shaanxi, China). The slides were dewaxed by rinsing with xylene followed by graded ethanol washes and then heated twice in $10 \mathrm{mmol} / \mathrm{l}$ sodium citrate $(\mathrm{pH}$ 6.0) for $5 \mathrm{~min}$ each in a microwave oven for antigen retrieval. Endogenous peroxidase activity was blocked in methanol containing $3 \%$ hydrogen peroxide for $10 \mathrm{~min}$ at room temperature. After washing 

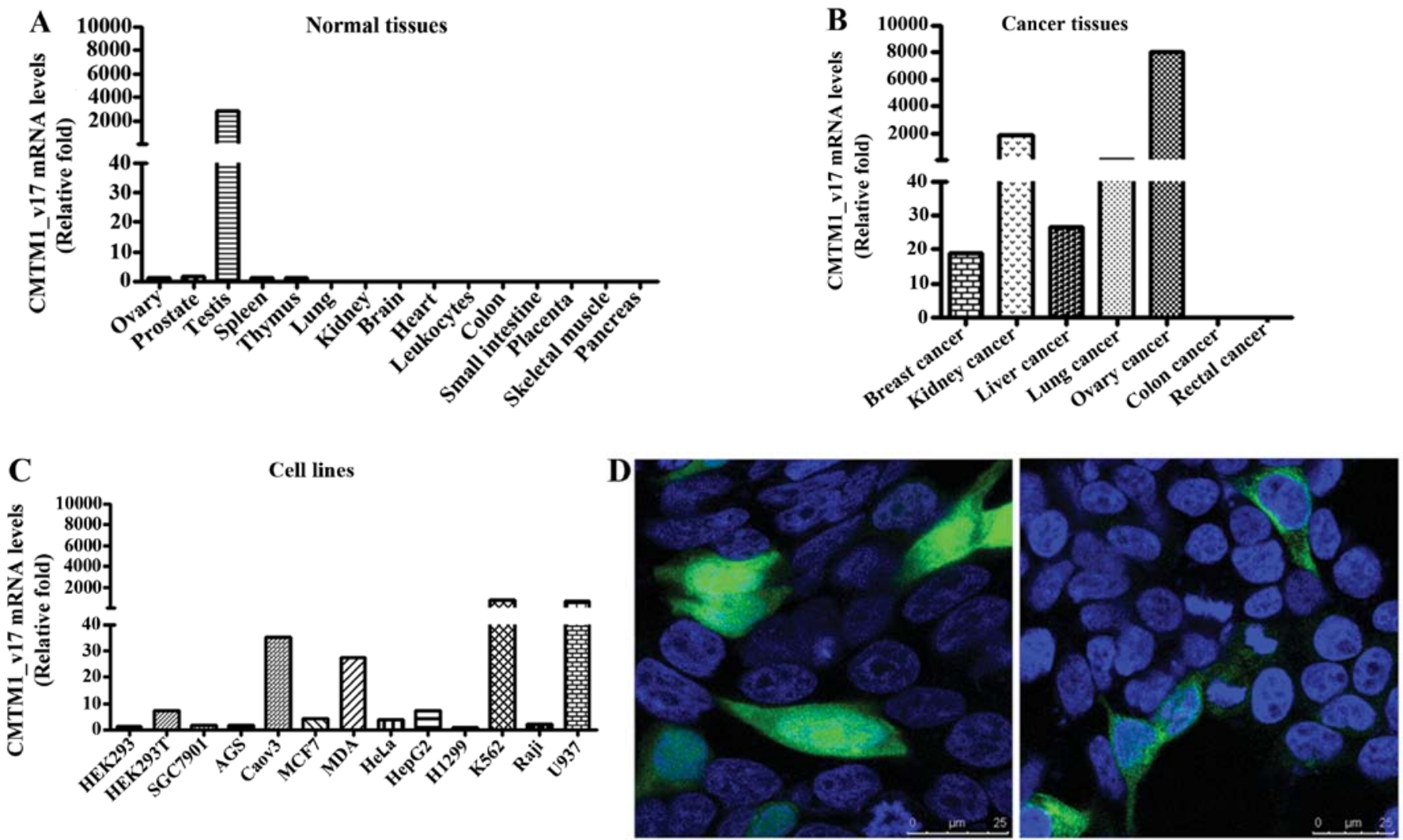

Control EGFP transfectants CMTM1_v17-EGFP transfectants

Figure 1. Relative expression profiles of $C M T M 1$ v17 mRNA and subcellular localization of $C M T M 1$ 1_v17. CMTM1_v17 mRNA levels are shown via real-time qPCR analysis in a panel of normal (A) and tumor (B) tissues and in a series of cell lines (C). CMTMI_v17 expression was normalized to GAPDH expression. (D) HEK293T cells were transiently transfected with CMTM1_v17 or a control EGFP vector. The subcellular localization of the fusion protein was determined by confocal microscopy.

with PBS, the slides were incubated at room temperature for 10 min in $10 \%$ normal goat serum/1X PBS. Then, the slides were incubated with rabbit polyclonal antibody to human CMTM1_v17 for $1 \mathrm{~h}$ at room temperature. A monoclonal anticancer antigen 15-3 (CA15-3) antibody (Zhongshan, Beijing, China) was used as a positive control. After adequate washing, they were subjected to HRP-labeled polymer for $30 \mathrm{~min}$ and $\mathrm{DAB}+$ substrate - chromogen solution for $5 \mathrm{~min}\left(\right.$ ChemMate $^{\mathrm{TM}}$ DAKO EnVision ${ }^{\mathrm{TM}}$ System; Dako, USA). All stained sections were counterstained with hematoxylin, then dehydrated and mounted with coverslips.

Cell growth analysis. Cell viability was assessed by trypan blue staining followed by counting of the unstained cells. Relative cell numbers were determined using the MTT colorimetric assay. Transfected cells were plated in 96-well plates at a density of 2,000 cells/well in $100 \mu \mathrm{l}$ medium. At the indicated time points, MTT solution was added and samples were incubated for $4 \mathrm{~h}$. After that, the absorbance was measured at a wavelength of $570 \mathrm{~nm}$. Each group was assayed in triplicate and each experiment was repeated three times.

Detection of phosphatidylserine externalization. At the indicated time points after transfection, the detached and adherent (trypsinized) cells were collected, washed twice with PBS and resuspended in $200 \mu \mathrm{l}$ Annexin $\mathrm{V}$ binding buffer (10 mM HEPES pH 7.4, $140 \mathrm{mM} \mathrm{NaCl}, 1 \mathrm{mM} \mathrm{MgCl}_{2}, 5 \mathrm{~m}$
$\mathrm{MKCl}, 2.5 \mathrm{mM} \mathrm{CaCl}{ }_{2}$ ). FITC-conjugated Annexin V was added to a final concentration of $0.5 \mu \mathrm{g} / \mathrm{ml}$. After incubation for $30 \mathrm{~min}$ at $4^{\circ} \mathrm{C}$ in the dark, propidium iodide (PI) was added to samples at $1 \mu \mathrm{g} / \mathrm{ml}$. Phosphatidylserine (PS) externalization analysis was performed on a FACSCalibur flow cytometer (Becton-Dickinson, USA).

Dual luciferase assay. Transient transfection of HEK293T cells was performed using VigoFect (Vigorous) according to the manufacturer's instructions. The relative luciferase activity was determined with a Dual-Luciferase Reporter Assay System (Promega) using a Veritas Microplate Luminometer (USA) by measuring firefly luciferase activity normalized by Renilla luciferase activity.

Statistical analysis. A Chi-square test was used to compare the expression of CMTM1_v17 between normal and tumor tissues. A p-value (two-sided) of 0.05 was considered to indicate a statistically significant result. Statistical analysis was performed using SigmaStat 2.03 software (SPSS, Inc.).

\section{Results}

Expression pattern of CMTM1_v17 mRNA. The expression pattern of human CMTM1_v17 mRNA transcripts was determined by real-time qPCR. CMTM1_v17 mRNA was highly expressed in testicular tissue, but was expressed at a 
Table I. Western blot analysis of CMTM1_v17 in breast cancer and non-cancerous mammary tissues.

\begin{tabular}{lccr}
\hline & Negative $\mathrm{n}(\%)$ & Positive $\mathrm{n}(\%)$ & P-value \\
\hline Non-cancerous mammary tissues $(\mathrm{n}=17)$ & $15(88.24)$ & $2(11.76)$ & $<0.01$ \\
Breast cancer tissues $(\mathrm{n}=20)$ & $7(35.00)$ & $13(65.00)$ & \\
\hline
\end{tabular}

Table II. Expression of CMTM1_v17 and CA15-3 in tissue microarray analysis.

A, Expression of CMTM1_v17 in tissue microarray analysis

\begin{tabular}{lccr}
\hline Samples & Weak staining $\mathrm{n}(\%)$ & Moderate/strong staining $\mathrm{n}(\%)$ & P-value \\
\hline Normal/non-cancerous $(\mathrm{n}=127)$ & $104(81.89)$ & $23(18.11)$ & $<0.01$ \\
Tumor $(\mathrm{n}=105)$ & $34(32.38)$ & $71(67.62)$ & \\
\hline
\end{tabular}

B, Expression of CA15-3 in tissue microarray analysis

\begin{tabular}{lccr}
\hline Samples & Faint staining n (\%) & Moderate/strong staining n (\%) & P-value \\
\hline Normal/non-cancerous (n=127) & $101(79.53)$ & $26(20.47)$ & $<0.01$ \\
Tumor $(\mathrm{n}=105)$ & $38(36.19)$ & $67(63.81)$ & \\
\hline
\end{tabular}

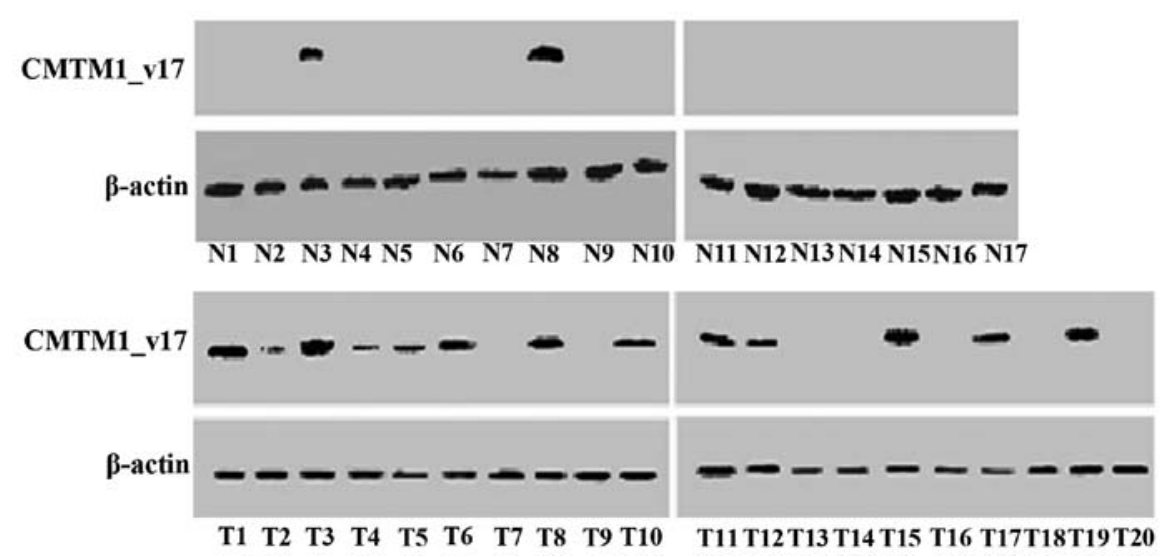

Figure 2. Expression of CMTM1_v17 protein in normal breast tissues and breast tumor tissues by immunoblotting. Normal tissues (n=17) are shown in lanes N1-N17 and tumor tissues $(n=20)$ are shown in lanes T1-T20.

low or undetectable level in other tissues (Fig. 1A). However, expression of CMTM1_v17 mRNA was detected in tumor tissues from breast, kidney, lung, liver and ovarian cancers, but not colon or rectal cancers (Fig. 1B). We also found that the expression level of CMTM1_v17 mRNA was high in K562 and U937 cells, moderate in MDA-MB-231 and Caov3, and relatively low in other cells lines (Fig. 1C).

Subcellular localization of CMTM1_v17. To investigate the subcellular localization of the human CMTM1-v17 protein, we examined the localization of the CMTM1_v17-EGFP construct using confocal fluorescence microscopy. In the cells that overexpressed the control EGFP vector, the fluorescence was distributed throughout the transfected cells with no specific subcellular localization while in the cells overexpressing
CMTM1_v17-EGFP, moderate fluorescence was detected only in the cytoplasm (Fig. 1D).

Expression of CMTM1_v17 is more prevalent in breast tumor than in normal breast tissues. CMTM1_v17 protein was detected in both breast tumor and adjacent non-cancerous mammary tissues by western blotting. In these samples, CMTM1_v17 expression was more prevalent in tumor than in normal breast tissues (Fig. 2). CMTM1_v17 was detected in $13 / 20(65.00 \%)$ tumors compared to only $2 / 17(11.76 \%)$ non-cancerous breast tissues (Table I). Immunohistochemistry was performed using commercially available tissue microarrays. Results showed that only 23/127 (18.11\%) normal breast or non-cancerous tissues expressed CMTM1_v17 at a high level. Most of the normal breast sections $(82 / 100,82.0 \%)$ 


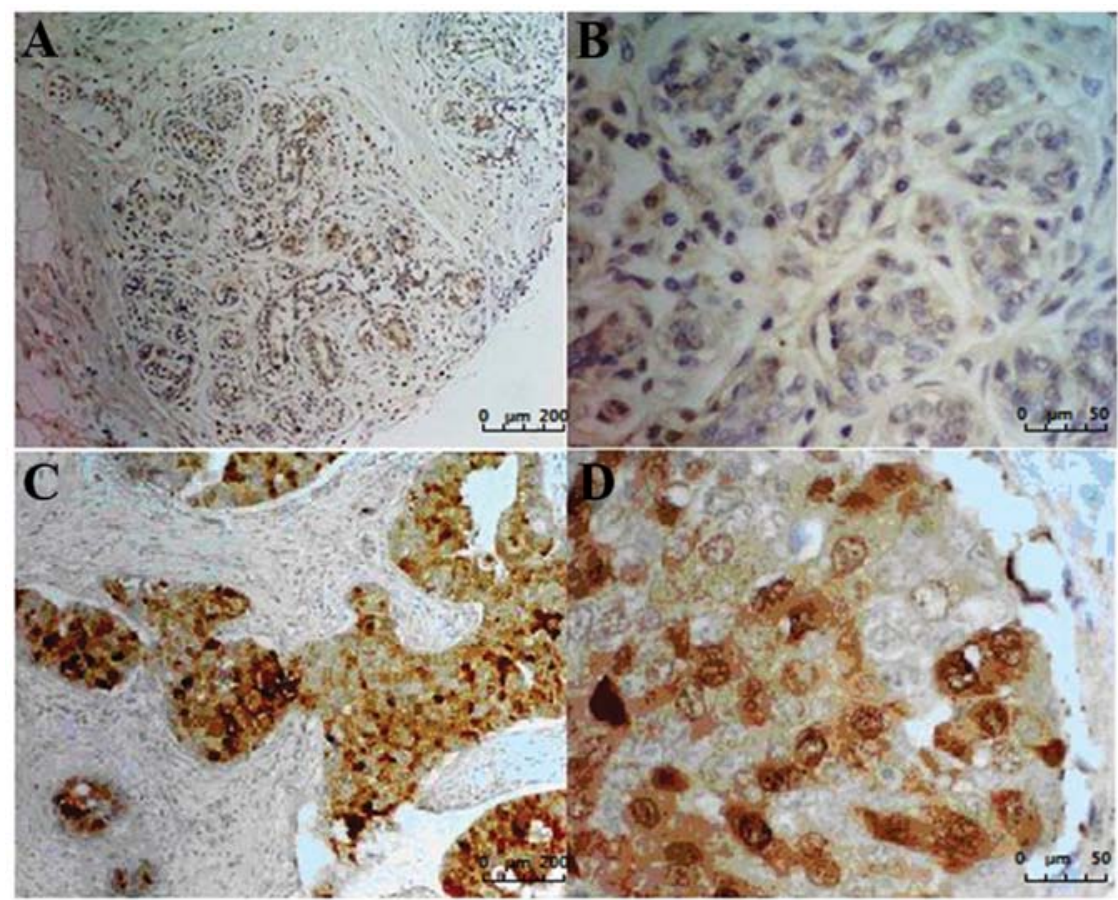

Figure 3. Analysis of CMTM1_v17 protein expression in normal breast and tumor tissues by immunohistochemistry. A tissue microarray containing 105 cases of breast cancer and 127 matched breast tissues (100 normal tissues and 27 non-cancerous tissues) was subjected to immunohistochemical staining for the expression of CMTM1_v17. Images of CMTM1_v17 protein expression are shown in normal tissues at x10 (A) or x40 (B) magnification and in tumor tissues at $\mathrm{x} 10$ (C) or $\mathrm{x} 40$ (D) magnification.

and non-cancerous breast tissues $(22 / 27,81.48 \%)$ showed only faint staining. Of the 105 tumor samples, $71(67.6 \%)$ showed moderate or strong expression of CMTM1_v17 (Fig. 3 and Table IIA). CA15-3 is a cell surface marker expressed by various tumor cells including breast cancer. The tissue microarray comprised of tumor $(\mathrm{n}=105)$ and normal/non-cancerous $(\mathrm{n}=127)$ tissues was subjected to immunohistochemical staining with anti-CA15-3 antibody. Approximately 67/105 (63.81\%) tumor and 26/127 (20.47\%) normal/non-cancerous tissues expressed CA15-3 (Table IIB). Table III shows the correlation analysis (McNemar's test, $\mathrm{p}>0.05$ ) between $C M T M 1 \_v 17$ and CA15-3 in normal/non-cancerous samples (Table IIIA) or in tumor samples (Table IIIB). The co-staining of CMTM1_v17 and CA15-3 increased the positive rate to $83.81 \%$.

CMTM1_v17 promotes the proliferation of MDA-MB-231 cells. The breast cancer cell line MDA-MB-231 was transfected with CMTM1_v17 or control vector and then the cells were assayed for proliferation. Results obtained from cell counting and MTT analysis showed that $C M T M 1_{-}$v17 had an obviously positive effect on the growth of MDA-MB-231 cells (Fig. 4).

CMTM1_v17 increases the resistance of MDA-MB-231 cells to $T N F-\alpha$-induced apoptosis. Since CMTM1_v17 promoted proliferation of MDA-MB-231 cells, we considered whether it also played a role in cellular apoptosis. To investigate this possibility, MDA-MB-231 cells were transiently transfected with CMTM1_v17 or control vector and stimulated with TNF- $\alpha$. The percentage of Annexin V-positive cells (15.95\%) in CMTM1_v17 overexpressing cells was much lower than that in cells transfected with the control vector $(23.30 \%)$ at $24 \mathrm{~h}$ (Fig. 5A). The result was more marked at $48 \mathrm{~h}$ (Fig. 5B).
Table III. Correlation analysis between CMTM1_v17 and CA15-3 in normal/non-cancerous and tumor samples.

A, Correlation analysis between CMTM1_v17 and CA15-3 in normal/non-cancerous samples (McNemar's test, $\mathrm{p}>0.05$ ).

CA15-3

Normal/non-cancerous

samples $(\mathrm{n}=127)$

Negative

Positive

CMTM1_v17

Negative

86

18

Positive

13

10

B, Correlation analysis between CMTM1_v17 and CA15-3 in tumor samples (McNemar's test, $\mathrm{p}>0.05$ ).

\begin{tabular}{lcc}
\hline & \multicolumn{2}{c}{ CA15-3 } \\
\cline { 2 - 3 } Tumor samples ( $\mathrm{n}=105)$ & Negative & Positive \\
\hline CMTM1_v17 & & \\
Negative & 17 & 18 \\
Positive & 20 & 50 \\
\hline
\end{tabular}

Similar results were obtained with human MCF-7 cells (data not shown).

Silencing of CMTM1_v17 expression sensitizes MDA-MB-231 cells to TNF- $\alpha$-induced apoptosis. We next investigated 

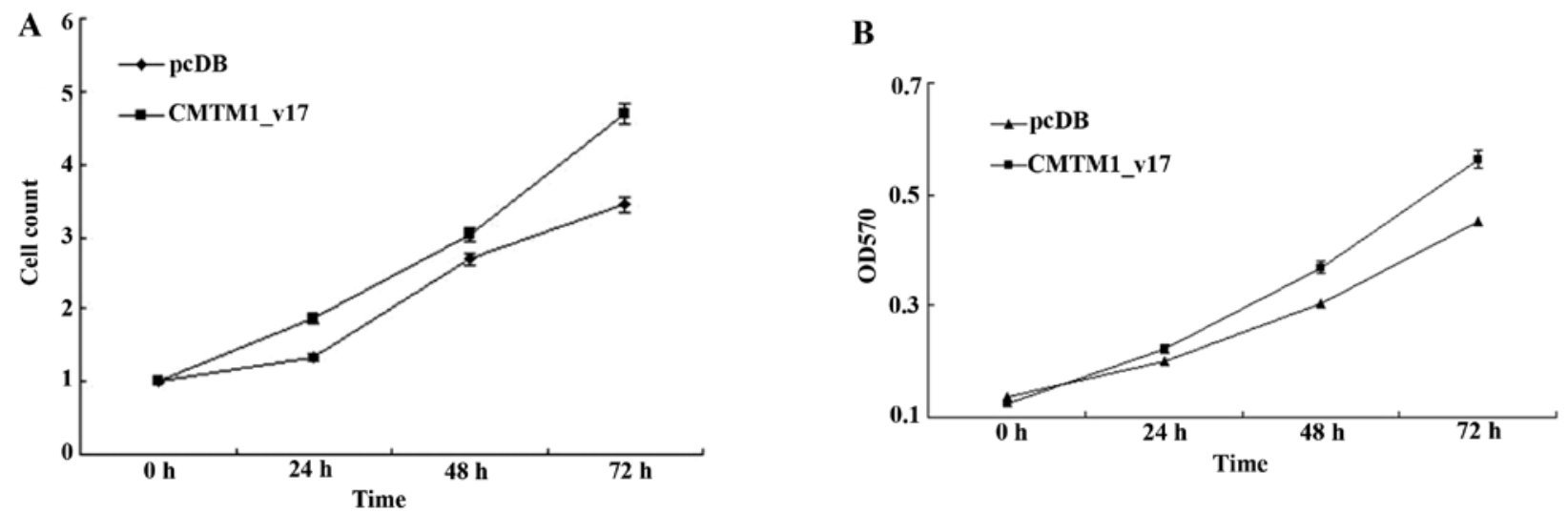

Figure 4. CMTM1_v17 promotes proliferation of MDA-MB-231 cells. MDA-MB-231 breast cancer cells were transiently transfected with CMTM1_v17 or a control vector pcDB. Over a 72 -h period, cell proliferation was determined at $24 \mathrm{~h}$ intervals by cell counting (A) and MTT assay (B).
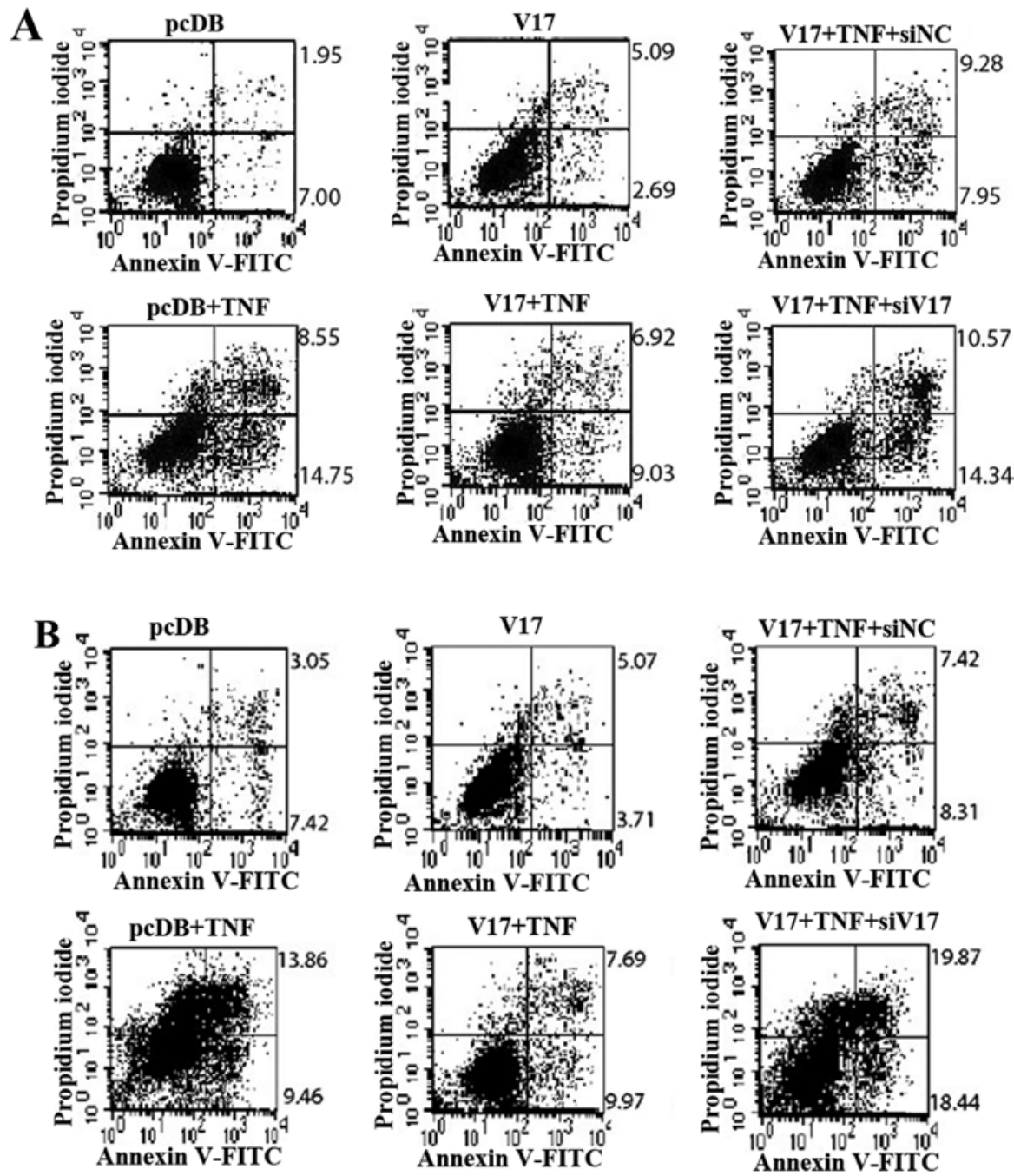

Figure 5. TNF- $\alpha$-induced apoptosis in breast cancer cells. MDA-MB-231 cells were transfected with CMTM1_v17 or pcDB and/or siRNA against $C M T M 1$ 1v17. These cells were treated with TNF- $\alpha(20 \mathrm{ng} / \mathrm{ml})$ and then assayed for apoptosis by FACS at $24 \mathrm{~h}(\mathrm{~A})$ and $48 \mathrm{~h}(\mathrm{~B})$.

whether the silencing of CMTM1_v17 expression sensitized MDA-MB-231 cells to TNF- $\alpha$-induced apoptosis. CMTM1-v17 expression was silenced using targeted siRNA and non-silencing siRNA as a control. Annexin V/PI staining was used to detect apoptotic cells after exposure to TNF- $\alpha$.
We found that siRNA-mediated silencing of CMTM1-v17 restored the sensitivity of MDA-MB-231 cells to TNF- $\alpha$ induced apoptosis (Fig. 5). These data further demonstrate that $C M T M 1$-v17 promotes cellular resistance to TNF- $\alpha$ induced apoptosis. 

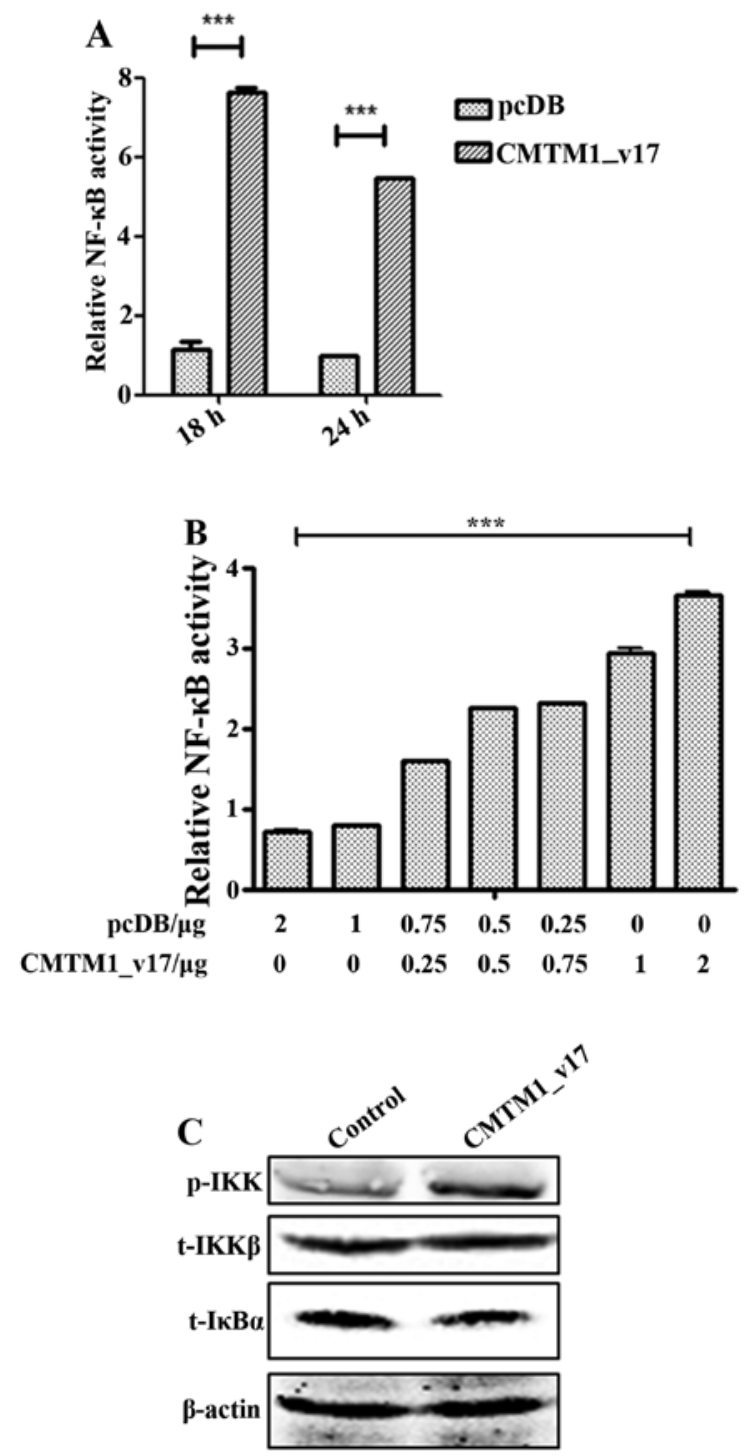

Figure 6. Overexpression of $C M T M 1$ v17 activates the NF-кB signaling pathway. HEK293T cells were co-transfected with CMTM1_v17 or a control vector, along with NF-кB-luciferase and control Renilla reporter plasmids. (A) At the indicated time points, the cells were harvested to measure NF- $\kappa \mathrm{B}$ induction by calculating the luciferase/Renilla ratio. (B) HEK293T cells were

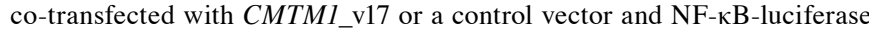
and control Renilla reporter plasmids at the indicated concentrations. After $18 \mathrm{~h}$, the cells were harvested and NF-kB induction was measured. (C) After overexpression of $C M T M 1$ _v17 for $12 \mathrm{~h}$, the expression of pIKK, total IKK $\beta$ and $\mathrm{I} \kappa \mathrm{B} \alpha$ was measured by immunoblotting of whole cell lysates.

CMTM1_v17 may function via activation of the NF- $\kappa B$ signaling pathway. To investigate the mechanisms underlying the enhanced proliferation and resistance to TNF- $\alpha$-induced apoptosis in cells overexpressing CMTM1_v17, we performed luciferase reporter assays in HEK293T cells for NF- $\mathrm{KB}$ activity by transiently transfecting pcDB or CMTM1_v17 with NF- $\kappa \mathrm{B}-l u c i f e r a s e$ and control Renilla reporter plasmids. As is shown, CMTM1_v17 significantly increased the transcriptional activity of NF- $\kappa \mathrm{B}$ in a time- (Fig. 6A) and dose- (Fig. 6B) dependent manner. These data suggest that overexpression of CMTM1_v17 plays a tonic effect on the activation of NF- $\mathrm{kB}$ signaling. To provide further experimental evidence, we performed western blot analysis to detect the key members of NF- $\mathrm{KB}$ signaling pathway. In cells that overexpressed
CMTM1_v17, we detected increased IKK phosphorylation and decreased expression of total I $\mathrm{I} B \alpha$. Total IKK $\beta$ and $\beta$-actin expression levels served as a loading control (Fig. 6C). Taken together, the data from luciferase assays and western blot analysis suggest that activation of the NF- $\mathrm{KB}$ pathway by CMTMI_v17 was likely mediated via phosphorylation of IKK and decreased levels of total IкB $\alpha$.

\section{Discussion}

In the present study, we found that CMTM1_v17 was specifically highly expressed in human testes, many human tumor tissues and cell lines but was largely undetectable in the other normal tissues tested. This was consistent with our previous study (6), in which northern blot analysis revealed that $C M T M 1$ was highly expressed in testicular tissue while it was hardly detectable in other normal tissues. The distinctive expression profile of CMTM1_v17 in human tissues, namely its high expression in multiple neoplastic tissues and cell lines and its absence in normal tissues except for the testes, suggests that the CMTM1_v17 may contribute to tumorigenesis by increasing cellular proliferation.

In recent years, it has became evident that breast cancer is one of the most common malignancies and a leading cause of mortality among women. The sporadic (non-inherited) breast cancer that constitutes $>90 \%$ of all breast cancers is a complex and heterogeneous disease at both the clinical and molecular levels. Several genetic aberrations and changes in gene expression have been shown to occur during malignant transformation, development and progression of breast cancers (15). TNF- $\alpha$ can induce multiple mechanisms to initiate apoptosis in many tumor cell lines and causes tumor necrosis in certain animal models by binding its membrane receptor TNF-R1 (16). Our findings showed that, compared to normal breast tissues, the expression of CMTM1_v17 was higher in breast tumors. Moreover, ectopic expression of CMTM1_v17 in breast cancer cells promoted the proliferation as well as the resistance to TNF- $\alpha$-induced apoptosis of breast cancer cell MDA-MB-231 while silenced CMTM1_v17 expression sensitized cells to TNF- $\alpha$-induced apoptosis. We hypothesized that the abnormal expression of CMTM1_v17 in breast cancer might have clinical applications; in particular, it might be a novel marker of diagnosis and a therapeutic target in breast cancer.

It is well known that NF- $\kappa B$ induces a variety of antiapoptotic factors (17). To provide further mechanism evidence, the dual-luciferase reporter assay and western blot analysis were performed and the results suggested that overexpression of CMTM1_v17 protein induced robust NF- $\kappa \mathrm{B}$ activation. Therefore, we demonstrated that CMTM1_v17 could promote the proliferation and lead to partial resistance to TNF- $\alpha$ induced apoptosis likely via activation of the NF- $\mathrm{kB}$ signaling pathway.

In summary, we found that $C M T M 1$ v17 was highly expressed in a variety of tumors including breast cancer. Overexpression of CMTM1_v17 in the cell line MDA-MB-231 promoted proliferation and enhanced the resistance to TNF- $\alpha$ induced apoptosis likely via activation of the NF- $\mathrm{KB}$ pathway. Moreover, silencing of CMTM1_v17 restored the sensitivity of the cells to TNF- $\alpha$-induced apoptosis. These data indicate that CMTM1_v17 is a novel potential therapeutic target in 
breast cancer and may play an important role in its diagnosis. CMTM1_v17 may also be a new cancer/testis antigen, as it is also highly expressed in human testes while it is almost undetectable in other normal human tissues. Further studies are required to confirm the therapeutic efficacy of targeting CMTM1_v17 in breast cancer.

\section{Acknowledgements}

This study was supported by grants from the National Natural Science Foundation of China (nos. 91129707 and 81172001).

\section{References}

1. Han W, Lou Y, Tang J, et al: Molecular cloning and characterization of chemokine-like factor 1 (CKLF1), a novel human cytokine with unique structure and potential chemotactic activity. Biochem J 357: 127-135, 2001.

2. Han W, Ding P, Xu M, et al: Identification of eight genes encoding chemokine-like factor superfamily members 1-8 (CKLFSF1-8) by in silico cloning and experimental validation. Genomics 81 : 609-617, 2003.

3. Xia D, Li X, Lou Y, et al: Overexpression of chemokine-like factor 2 promotes the proliferation and survival of $\mathrm{C} 2 \mathrm{C} 12$ skeletal muscle cells. Biochim Biophys Acta 1591: 163-173, 2002.

4. Lou Y, Xia D, Han W, et al: Molecular cloning and characterization of rat chemokine-like factor 1 and 2. Gene 307: 125-132, 2003.

5. Rui M, Xia D, Zhang Y, et al: Molecular cloning and characterization of four isoforms of mCKLF, mouse homologues of human chemokine-like factor. Mol Biol Rep 30: 229-237, 2003.

6. Wang L, Wu C, Zheng Y, et al: Molecular cloning and characterization of chemokine-like factor super family member 1 (CKLFSF1), a novel human gene with at least 23 alternative splicing isoforms in testis tissue. Int J Biochem Cell Biol 36: 1492-1501, 2004.
7. Di Meo S, Airoldi I, Sorrentino C, Zorzoli A, Esposito S and Di Carlo E: Interleukin-30 expression in prostate cancer and its draining lymph nodes correlates with advanced grade and stage. Clin Cancer Res 20: 585-594, 2014.

8. Shi S, Rui M, Han W, et al: CKLFSF2 is highly expressed in testis and can be secreted into the seminiferous tubules. Int $\mathrm{J}$ Biochem Cell Biol 37: 1633-1640, 2005.

9. Jin C, Ding P, Wang Y and Ma D: Regulation of EGF receptor signaling by the MARVEL domain-containing protein CKLFSF8. FEBS Lett 579: 6375-6382, 2005.

10. Zhong J, Wang Y, Qiu X, et al: Characterization and expression profile of CMTM3/CKLFSF3. J Biochem Mol Biol 39: 537-545, 2006.

11. Plate M, Li T, Wang Y, et al: Identification and characterization of CMTM4, a novel gene with inhibitory effects on HeLa cell growth through inducing $\mathrm{G} 2 / \mathrm{M}$ phase accumulation. Mol Cells 29: 355-361, 2010.

12. Shao L, Cui Y, Li H, et al: CMTM5 exhibits tumor suppressor activities and is frequently silenced by methylation in carcinoma cell lines. Clin Cancer Res 13: 5756-5762, 2007.

13. Wang Y, Li J, Cui Y, et al: CMTM3, located at the critical tumor suppressor locus 16q22.1, is silenced by CpG methylation in carcinomas and inhibits tumor cell growth through inducing apoptosis. Cancer Res 69: 5194-5201, 2009.

14. Li H, Li J, Su Y, et al: A novel 3p22.3 gene CMTM7 represses oncogenic EGFR signaling and inhibits cancer cell growth. Oncogene 33: 3109-3118, 2014.

15. Liu JC, Voisin V, Bader GD, et al: Seventeen-gene signature from enriched Her2/Neu mammary tumor-initiating cells predicts clinical outcome for human HER $2^{+}: E R \alpha^{-}$breast cancer. Proc Natl Acad Sci USA 109: 5832-5837, 2012.

16. Locksley RM, Killeen N and Lenardo MJ: The TNF and TNF receptor superfamilies: integrating mammalian biology. Cell 104: 487-501, 2001.

17. Baldwin AS: Regulation of cell death and autophagy by IKK and NF- $\kappa \mathrm{B}$ : critical mechanisms in immune function and cancer. Immunol Rev 246: 327-345, 2012. 